\title{
Editorial: mobile data management and analytics
}

\section{Takahiro Hara ${ }^{1} \cdot$ Wang-Chein Lee ${ }^{2} \cdot$ Bin Yang $^{3}$}

Published online: 31 October 2019

(C) Springer Science+Business Media, LLC, part of Springer Nature 2019

This special issue of the GeoInformatica journal covers recent advances in data management and analytics in mobile, ubiquitous and pervasive computing. It contains four articles, which are extensions of the best research papers presented at the 19th IEEE International Conference on Mobile Data Management (MDM 2018) in Aalborg, Denmark. The MDM conference is a premier and prestigious forum for the presentation of high-impact research and exchange of innovative and significant ideas in the area of mobile data management and analytics. These papers went through several rounds of rigorous reviews by the guest editors and invited reviewers.

The paper, by Abdullah Sawas, Abdullah Abuolaim, Mahmoud Abuolaim, and Manos Papagelis, "A Versatile Computational Framework for Group Pattern Mining of Pedestrian Trajectories," presents a versatile method to mine group patterns from pedestrian trajectories that are obtained from motion videos. The proposed method is able to efficiently discover pedestrian groups according to different pattern semantics, which is able to accommodate a wide variety of application scenarios.

Postdiction, unlike prediction that predicts future values, aims at recovering past values which do not exist anymore, e.g., those deleted already due to storage limitation. The paper, by Constantinos Costa, Andreas Konstantinidis, Andreas Charalampous, Demetrios ZeinalipourYazti, and Mohamed F. Mokbel, "Continuous Decaying of Telco Big Data with Data Postdiction," presents two novel decaying operators for telco big data postdiction. Their

Bin Yang

byang@cs.aau.dk

Takahiro Hara

hara@ist.osaka-u.ac.jp

Wang-Chein Lee

wlee@cse.psu.edu

1 Department of Multimedia Engineering, Osaka University, Osaka, Japan

2 Department of Computer Science and Engineering, The Pennsylvania State University, State College, PA, USA

3 Department of Computer Science, Aalborg University, Aalborg, Denmark 
proposal employs machine learning models to abstract telco big data into compact models which is later used to recover past values. The proposal also supports streaming data.

Ridesharing is becoming increasingly important in urban transportation. Xiaoyi $\mathrm{Fu}, \mathrm{Ce}$ Zhang, Hua Lu, and Jianliang Xu proposed an new method to match requests and offers by considering both social comfort constraints and spatio-temporal constraints in their paper "Efficient Matching of Offers and Requests in Social-Aware Ridesharing." The problem is proved to be NP-hard and various pruning strategies are designed to reduce the search space which improves the efficiency.

Finally, the paper "Efficient Framework for Processing Top-k Queries with Replication in Mobile Ad Hoc Networks" considers top-k query processing in MANETs. Yuya Sasaki, Takahiro Hara, and Yoshiharu Ishikawa propose a topology-free data replication strategy to improve the efficiency while making sure the exactness of the query results.

These papers represent some of the different directions in the fast-growing area of mobile data management and analytics. We hope that these papers will inspire more research in this exciting area.

Publisher's note Springer Nature remains neutral with regard to jurisdictional claims in published maps and institutional affiliations. 